\title{
Technical Aspects of Surface Electromyography for Clinicians
}

\author{
Andrea Merlo* and Isabella Campanini
}

LAM - Movement Analysis Lab, Rehabilitation Department, Reggio Emilia Local Health Unit, Correggio, Reggio Emilia, Italy

\begin{abstract}
Surface electromyography (sEMG) is a measure of the electrical potential present on the skin in consequence of a muscle contraction. This voltage is detected by electrodes placed on the skin. Under certain limitations, when a proper protocol for electrode placement is used, the voltage measured on the skin can be related to the activity of a single specific muscle. The signal is represented as a trace that develops during time, increasing from zero up to tenths or hundreds of microvolts as the muscle becomes activated.

Being a non invasive and painless measure, sEMG has been applied in motion analysis to assess superficial muscle function, with application in sports, ergonomics, occupational and rehabilitation medicine. It allows for investigation of both muscle activation and muscle physiological characteristics.

The first part of this work provides an overview on sEMG signal acquisition technique and its representation, focusing on those elements that ensure signal reliability and on the sources of errors and artifacts. The second part of this work provides a critical overview on the information that can be extracted from the sEMG signal, dealing with amplitude, timing and morphology.
\end{abstract}

Keywords: Surface electromyography, muscle, technique, sEMG, crosstalk.

\section{ORIGIN OF THE SEMG SIGNAL}

When a muscle is activated by the central nervous system (CNS), muscle fiber contraction takes place, following the depolarization of the outer muscle-fiber membrane. The depolarized zone arises at the innervation zone and travels outwards the tendon regions. The depolarized zones of the fibers of each recruited motor unit (MU) give rise to an electrical field and generate voltage contributions that add up on the skin to form a voltage distribution. This sum is weighted by the distance of each source from the skin, thus the contribution to the skin potential of superficial MUs is higher than the contribution of deep MUs. By placing electrodes over the skin, the voltage in those points (areas) covered by the electrode can be measured. Typically, two electrodes per muscle are used and the difference between the two cutaneous potentials is acquired, as described below.

\section{THE VOLUME CONDUCTOR}

The biological tissues separating the sources and the detecting electrodes are referred to as volume conductor and their characteristics strongly affect the detected signal [1]. The cutaneous voltage distribution is reduced in amplitude, smoothed and "blurred", thus leading to a differential sEMG signal lower in amplitude and smoothed with respect to the signal that could be detected within the muscle, by needle or fine-wire EMG (Fig. 1) [1]. In this paper we will address only sEMG detected by means of a couple of electrodes. Many efforts to develop better detection system have been made in the last 20 years. Electrodes with different contact

*Address correspondence to this author at the Movement Analysis Lab, Rehabilitation Department, Reggio Emilia Local Health Unit, Correggio, Reggio Emilia, Italy; Tel: +39 0522 630391; Fax: +39 027005929 83; E-mail: andrea.merlo@ausl.re.it areas allow the detection of surface signal near in shape to the one detected by inserted needles such as the IB2 electrode $[2,3]$ and the concentric ring electrode developed by Farina and colleagues [4]. Electrode arrays have been also developed to assess muscle physiological properties $[5,6]$.

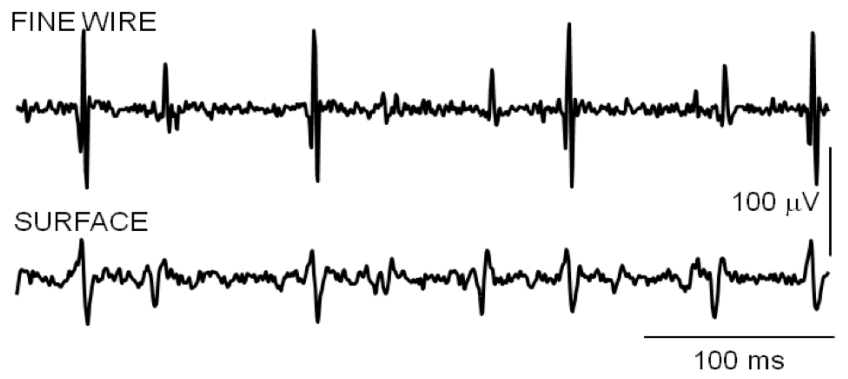

Fig. (1). Fine-wire and surface EMG detected from the brachioradialis muscle of a healthy subject. Surface potentials are smoothed and reduced in amplitude by the filtering effect of the subcutaneous tissues. Surface electrode diameter $2.5 \mathrm{~mm}$. Interelectrode distance $10 \mathrm{~mm}$.

We can consider the volume conductor that surrounds a source as consisting of the resting part of the muscle, the soft tissues, the fat layer and the skin layers. Conductor volume acts as a low-pass filter on the EMG signal. Each motor unit action potential becomes wider, smoothed and lower in amplitude. The thicker the volume conductor (fat layer, edema) the lower will be the sEMG signal amplitude. In practice, sEMG is not an adequate tool for assessing muscle function when a few centimeters of fat tissue are present in the investigated body region [3].

\section{DIFFERENTIAL DETECTION}

The membrane potential in the depolarization zone has the well-known shape described in physiology books, with a 
resting potential of 70/90 $\mathrm{mV}$ (negative inside the cell with respect to the extracellular environment). The depolarized zone arises at the neuro-muscular junction level and travels outwards the tendon regions. If surface electrodes are placed on the skin parallel to the muscle fibers, then the travelling potential is detected by the electrodes with a temporal delay that depends on the muscle fiber conduction velocity. Electrodes are connected to an electronic device referred to as differential amplifier. This device has two inputs and one output. The output is a potential that is the difference between the two input (electrode) potentials. Fig. (2) shows the generation of an action potential at the output of the differential amplifier when a single motor unit potential (MUAP) travels under the skin. Due to the differentiation process, the output waveform becomes biphasic.

The terms bipolar detection, differential detection and single differential detection are synonyms and refers to the use of two adjacent electrodes placed on the skin and connected to a differential amplifier.

The differential detection technique has the great advantage of deleting potentials that are equal under the electrodes, such as signals coming from far muscles that are very smoothed by the interposed tissues and similar over the skin in the electrode area, and the $50 / 60 \mathrm{~Hz}$ potential present on the whole skin, as induced by the electrical filed produced by the power line cables.

\section{THE EASE OF GETTING A SIGNAL}

It is useful to consider that a signal that looks like a properly acquired sEMG can be obtained by placing two electrodes on two different muscles (e.g. masseter and biceps brachii). When one of these two muscles is activated the signal on the screen increases because a voltage difference arises between the two locations, without providing any information on which of the two muscles is active. Similarly, it is sufficient to place two electrodes on a muscle to get a signal that increases in amplitude when some potential appears under one electrode. To prevent misplacements that could lead to clinical misinterpretation and to promote interoperator reliability, several protocols for electrode placement have been developed and purposed, that relate the presence of sEMG signal to the activity of a specific target muscle [79].

\section{THE SKIN-ELECTRODE INTERFACE}

Disposable electrodes typically used for sEMG detection during motion are made out of a metallic part in $\mathrm{Ag}-\mathrm{AgCl}$ (which is the electrode), covered by an adhesive conductive gel (which is the electrolyte) and surrounded by a plastic cap that is adhesive in the lower part. The electrode pick-up area is the whole gelled region that touches the skin.

From an electrical point of view, the skin-electrode complex is characterized by an impedance value. The lower

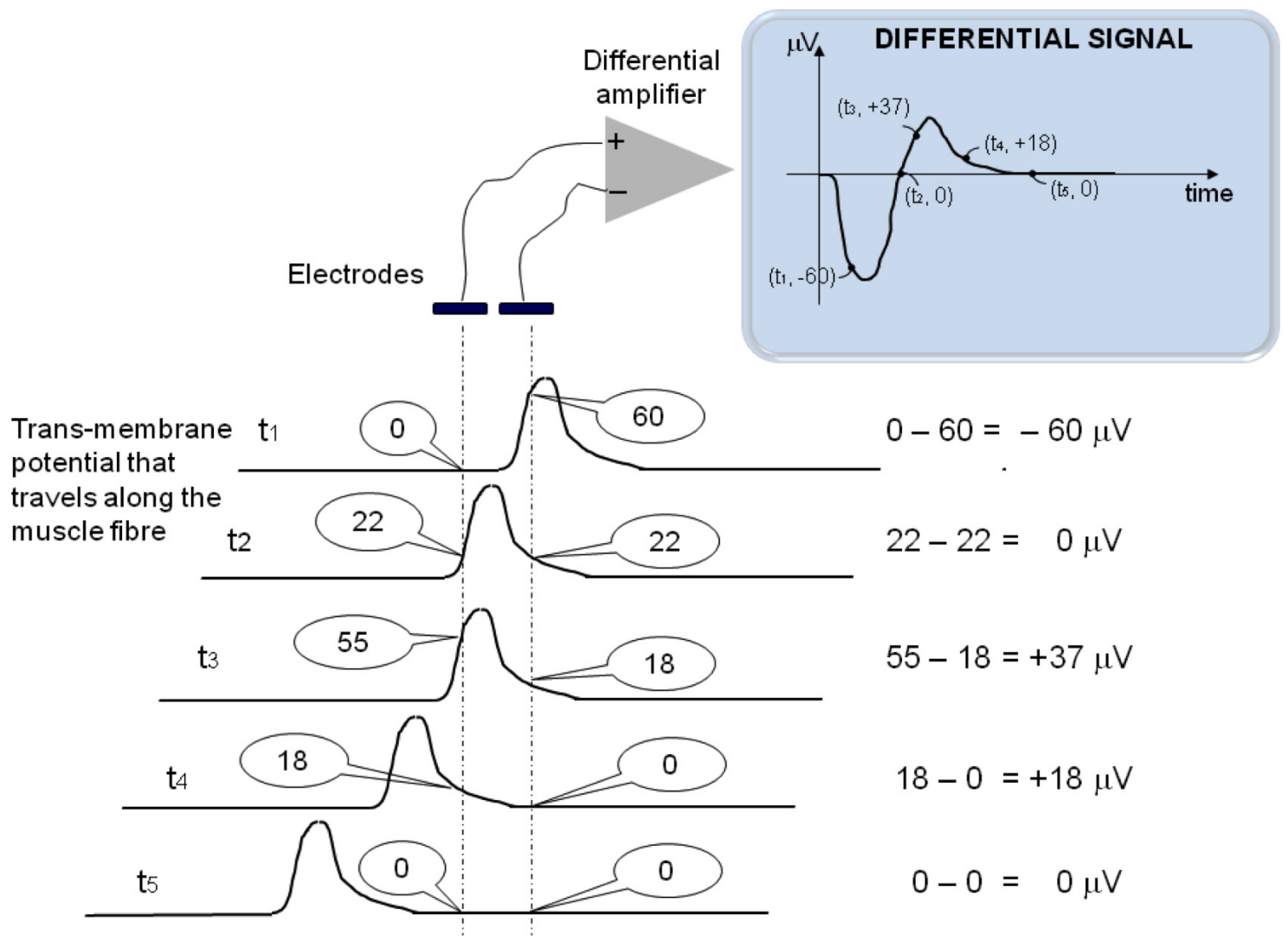

Fig. (2). A transmenbrane potential that travels along a muscle fiber, from the right to the left, is represented in five consecutive time instants in the lower part of the figure. Two electrodes are virtually placed on the muscle fiber and the electrode potentials at each time instant are reported in the callouts. The differential amplifier computes the difference between the electrode potentials (right column), thus producing the differential signal displayed by the electromyograph. Due to the differentiation process, the output waveform becomes biphasic. 
this impedance, the better is the signal propagation from the skin to the amplifier. Thus, lowering the skin impedance will result in a better signal detection. The skin-electrode complex impedance mainly depends on the top layer of the epidermis, the stratum corneum, which is dehydrated, lipophilic and, in conclusion, electrically insulated. Electrode impedance for different skin preparations was studied in [10]. Mildly rubbing the skin with medical abrasive paste is the best treatment to reduce electrode-skin impedance (about $-90 \%$ ). The widely used skin treatments with alcohol, ether or acetone are less effective, with a skin impedance reduction of about $-45 \%$ [10].

\section{MOTION ARTIFACTS}

Currents flowing into the body are due to the shift of ions, such as $\mathrm{Ca}^{++}, \mathrm{K}^{+}, \mathrm{Cl}^{-}$. Currents flowing through the wires of the electromyograph are due to the flow of electrons. Thus, a transduction must take place at the skinelectrode interface. At both the electrode-electrolyte interface and the electrolyte-skin interface a double layer of charge forms, as described in [11], that determines offset voltages to appear at the interfaces. If an electrode is moved or the skin is stretched, this movement disturbs the distribution of charge at the interface and results in a large differentials signal detected by the electrode pair. This disturb is referred to as motion artifact. Any relative movement between skin and electrolyte and between electrolyte and electrode lead to an artifact in the detected sEMG signal. The shape of a movement artifact is shown in Fig. (3).

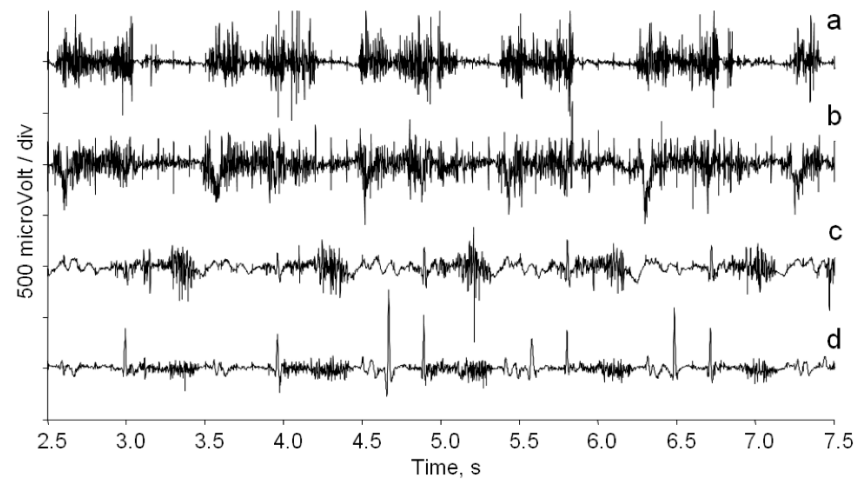

Fig. (3). sEMG traces variously affected by motion artifacts: a) good signal with a minimum drift of the baseline; b) signal with periodic baseline fluctuations, as at $\mathrm{t}=3.5 \mathrm{~s}$; c) signal with motion artifacts; d) signal with narrow motion artifacts. Raw unfiltered data are presented. Electrode size: $18 \mathrm{~mm}$, interelectrode distance: $20 \mathrm{~mm}$.

Movement artifacts can be frequent in sEMG signals when 1) electrode adhesion is poor and contact is not perfectly stable, 2) adhesive tape is placed over the electrodes, 3) skin is not properly prepared, 4) cables hit the electrodes (e.g. at foot contact during gait), 5) preamplifiers have stiff cables that deliver a tension to electrodes during motion, 6) electrodes collide due to skin displacement near joints with a high range of motion (e.g. vastus medialis during gait).

Motion artifact can be significantly reduced by removing the stratum corneum, as demonstrated in [12].
It is important to consider that, in raw data, the signal produced by a movement artifact is completely different from that produced by muscle activity, thus being detectable. Conversely, movement artifact could be non detectable, by eyes, in filtered data (see below) and lead to misinterpretation. In the author's experience, the practice of looking at filtered data commonly adopted by clinicians working in gait laboratories may lead them to consider rhythmic short activity bursts present in one muscle trace as a stretch-reflex generated activity, while being due the collision of cables against one electrode at each heel strike when an abrupt leg deceleration takes place.

As a general recommendation, the operator should look at raw data after each acquisition. If artifacts are present in the signal, they can be eliminated by either replacing electrodes, removing the skin corneum stratum or modifying the cable configuration.

\section{CROSSTALK}

The depolarized zone can be modeled as a current tripole: a central current sink, where the positive ions enter into the fiber, and two lateral current sources, where the ions start moving towards the sink. This tripole determines the appearance of the electrical field that reflects muscle activity we are interested in. The depolarized zone travels from the neuro-muscular junction towards the tendon regions. Tendons have no ion to deliver to the depolarized zone, so the travelling current tripole is converted to a dipole localized at the end of the muscle fiber, before being extinguished. The electric field produced by this dipole is totally different from the one produced by the travelling tripole, being especially less reduced by distance. That is, as the distance from the active fiber increases the potential produced by the dipole overcomes the one produced by the tripole. While the contribution due to travelling potentials can be measured only in a restricted area of the skin above the active motor unit, the contribution due to end-fiber potentials can be detected even on the skin of adjacent inactive muscles. If this happens, electrodes placed on an inactive muscle would provide a misleading active-like sEMG signal. This phenomenon is referred to as crosstalk and represents the main risk in the clinical use of surface EMG, because most of the clinically relevant information is related to the on/off of the muscle activity that is altered by crosstalk.

As closeness to tendons increases the risk for crosstalk, superficial muscles that are near to wide tendon regions (such as the vasti) or near to the insertion of muscle groups (such as at the epitroclea for the fore-arm extensors) will be mainly affected by the end-fiber effect. Similarly, the signal detected from the soleus muscle by an electrode placed over the Achilles tendon will be a mix of calf muscle activity, instead of the signal of the soleus itself, as detectable from the medial aspect of the leg. The dipole-generated crosstalk cannot be reduced by either minimizing the distance between the detecting electrodes (see below) or high-pass filtering the acquired data.

Crosstalk can be also present if the electrode pair is improperly placed too near to a neighbor muscle or partially above it [13]. In this case, the tripole-generated potential of the neighbor muscle adds to the one of the target muscle. 
The risk for such error is supported by the dimension of most of the commercially available disposable electrodes, which are about 2 centimeters in width or diameter and can thus be wider than the section of the target muscle (e.g. peronei muscles, fore-arm muscles, child muscles). The tripolegenerated crosstalk can be minimized by reducing the distance between the center of the electrodes (see below).

Placing the electrodes on the muscle belly does not generally protect against the risk of crosstalk. A list of minimum crosstalk areas for sEMG detection has been published in $[7,14]$ for electrodes of $2.5 \mathrm{~mm}$ in diameter placed at a distance of $10 \mathrm{~mm}$. In Fig. (4) crosstalk on the tibialis anterior of a child coming from plantar flexor muscle is visible. sEMG was detected by using electrodes of $20 \mathrm{~mm}$ in diameter.

No signal processing technique for crosstalk identification and removal is currently available to clinicians in their daily routine. In literature, recent attempts can be found in $[15,16]$.
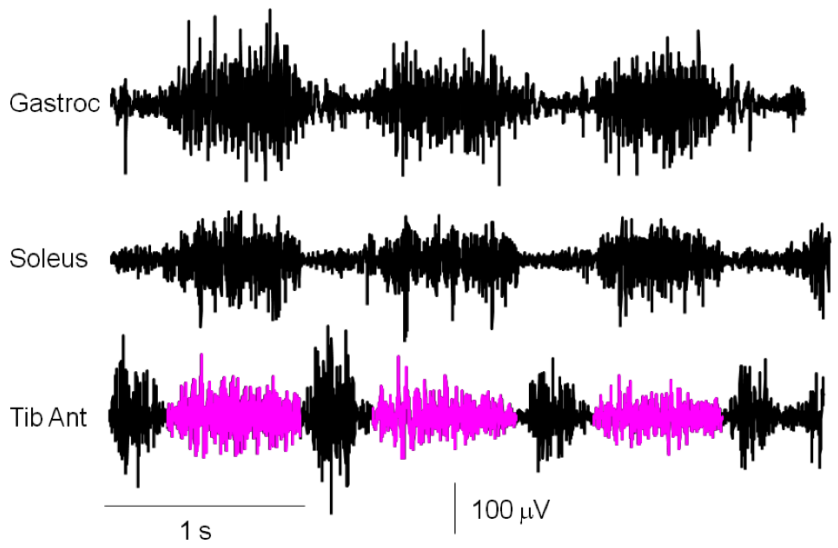

Fig. (4). sEMG signals acquired during gait from the leg of 10 years old kid, by means of $18 \mathrm{~mm}$ width electrodes placed at an interelectrode distance (center to center) of $20 \mathrm{~mm}$. Crosstalk from plantar flexors is outlined in purple.

\section{ELECTRODE SIZE AND INTERELECTRODE DISTANCE}

Electrode area influences the sEMG amplitude. The wider the area, the higher the amplitude. Again, the wider the area, the higher the distance at which electrodes can be placed without superimposition. The distance between electrodes is referred to as the interelectrode distance (IED). The higher the IED, the wider the detection volume and consequently the detected amplitude. The main drawback in the use of wide electrodes is the increase of the detected volume, that is the region from which potentials are detected, with a corresponding increase in the risk of crosstalk. In general large electrodes may either directly (due to superimposition) or in-directly (due to crosstalk) pick-up signals from neighbouring muscles. The effect of interelectrode distance on sEMG detection is widely addressed in [1].

In accordance with the recommendations produced by the European Concerted Action Surface EMG for noninvasive assessment of muscles [8,9], electrode diameter has to be lower than $10 \mathrm{~mm}$, and the distance between electrode centers should be less or equal to $20 \mathrm{~mm}$. Smaller electrodes have to be used on muscle whose section is lower than or near to $10 \mathrm{~mm}$.

\section{THE ELECTROMYOGRAPH}

An electromyograph is basically made out of four components: the pre-amplifier, the transmitter-receiver couple, the hardware filters and the analog to digital (A/D) converter.

The pre-amplifier is typically contained in a small box near to the electrodes. It is powered by either internal batteries or the connection cable, computes the difference between the two electrode potentials (it is the differential amplifier) and amplifies the obtained differential signal (e.g. $x$ 1000) in order to send it through the cable or the air to the $\mathrm{A} / \mathrm{D}$ converter.

The transmission system varies from producer to producer, according to the field of application of the device and the technology used. Cable connected preamplifiers are wired to a small wearable unit that can either store data or send them to a computer by means of a cable or a wireless transmission.

Hardware filters are used to remove unwanted components from the signal before the A/D conversion. Typically, a high pass filter at a very low frequency, such as $10 \mathrm{~Hz}$, is used to remove the baseline drift and, in part, motion artifacts. In the author's opinion, it is better to acquire raw data and filter them afterwards, if necessary, via software. A low pass filter is normally present before the $\mathrm{A} / \mathrm{D}$ block. It removes higher frequency contributions that are produced by electronic components and do not rely on the EMG signal, such as frequencies higher than $400 \mathrm{~Hz}$. These contributions are, in general, negligible. For the use of EMG in motion analysis, the value of the cut-off frequency of the low-pass filter can be set, with no noticeable differences, at $300 \mathrm{~Hz}$ or more.

According to the approach used by the manufacturer, hardware filters can be both user-modifiable or fixed. Similarly, a second amplification stage indicated with "gain" can be present to further amplify data before the A/D conversion. Systems that allow the user to modify filters and gain are more flexible, but require a certain degree of user knowledge.

The $\mathrm{A} / \mathrm{D}$ conversion is the process that transforms voltages into numbers for the computer. The amplification and $A / D$ conversion process can be compared to the process of taking a photograph using a digital camera. In the camera the screen we see indicates the borders of the available input area. The image within these borders will be captured. Similarly, the A/D converter has an input range, in Volts, the limits of which indicate the maximum and minimum detectable voltage. Typical values are $\pm 5 \mathrm{~V}$ or $\pm 2.5 \mathrm{~V}$.

In the camera, we use the zoom to enlarge the target object we are interested in until it fills the screen of the camera. If the object is small, we need to zoom on it to fit the screen. If we zoom too much, part of the object will be lost. In the electromyography we amplify the signal detected on the skin to fill the input range. The skin potential is low in amplitude (hundred of microvolts) and we need to multiply it by 1000 or 5000 or more, until is nearly fills the A/D converter input range. If we amplify too much, the part of 
the signal that exceeds the input range (e.g. 5V) will be lost. If we consider the input range of the $\mathrm{A} / \mathrm{D}$ convertor to be similar to the size of the screen of the camera, the best procedure is to amplify the signal (to zoom the object) until it fits the $\mathrm{A} / \mathrm{D}$ input range (the screen size), in order to maximize the resolution of the stored signal (image).

Several commercially available devices are designed for the use with pre-gelled $2-\mathrm{cm}$ wide electrodes and have a gain of 1000 or 5000 , because they are expected to amplify signals ranging from a few hundreds of microvolts to a few millivolts (e.g. during maximum contractions in athletes).

In order to convert the image colors into numbers, the camera has a square or rectangular sensor, which is divided into row and columns as in a matrix. Each dot in the sensor matrix will produce a number that expresses the image color in that dot. The higher the number of rows and columns in the sensor, the higher the camera resolution. Similarly, the A/D converter can be thought as a grid that covers one second of the EMG signal. The EMG will be converted in a sequence of numbers that are the coordinates of each dot of the grid the EMG trace passes through. This values are times and EMG values (in volts) at those times.

In the $A / D$ conversion grid, the number of vertical columns is determined by the sampling frequency and the number of horizontal rows is determined by the number of bits of the A/D. The higher the sampling frequency, the higher the number of samples per second taken from the analogue EMG signal. A sampling frequency of $1000 \mathrm{~Hz}$ indicates that one second of EMG will be represented by 1000 numerical values. Sampling frequency of $1000 \mathrm{~Hz}$ or $2000 \mathrm{~Hz}$ are adequate for surface EMG acquisition [17].

The number of rows of the A/D converter depends on its number of bits. An EMG device with 12 bits has $2^{12}=4096$ levels or rows; an EMG device with 16 bits has $2^{16}=65536$ levels or rows. These levels are uniformly distributed in the input range of the A/D converter. For example, $a \pm 2.5 \mathrm{~V} \mathrm{~A} / \mathrm{D}$ with an input range of 12 bits has a resolution of 5/4096= $0.0012 \mathrm{~V}=1.2 \mathrm{mV}$. If the gain is set to 1000 , then the resolution at the skin level becomes 1.2 microvolts.

An indicator of the minimum detectable voltage of a device is the input referred noise voltage, expressed in $\mathrm{V}_{\mathrm{rms}}$ and reported by the device manual. The lower this value, the better the device. Electromyographs with input referred noise voltage of about $1-2 \mu \mathrm{V}_{\text {rms }}$ are available, corresponding to a 5-7 $\mu \mathrm{V}$ peak to peak noise generated baseline. A user can simply test the noise level of his device by shortcutting two electrodes (that is putting the two gelled areas together) and measuring the peak to peak amplitude of the baseline.

In practice, it is important to remember that the smaller the electrodes we use, the lower will be the detected potential, thus requiring high amplification (e.g. 10000) to gain resolution.

In fixed-gain devices a number of bits as high as 16 or more is preferable, if we plan to work with miniaturized electrodes. Instead of amplifying the signal to fit the screen, we gain resolution by increasing the number of rows in the sensor and zoom on the stored signal afterward, via software. The higher the number of bits, the more we can zoom on the acquired signal.

\section{DATA ON THE SCREEN}

A sEMG plot is a graph of the differential voltage detected by the electrode pair plotted versus time. In motion analysis, several muscles are plotted together in a unique graph. It is mandatory to set a common $\mathrm{Y}$-axis scale before looking at data, to prevent misinterpretations that could adversely influence clinical decision.

We recommend that clinicians who use sEMG devices check for the $y$-axis setting in the software and remove, if present, the autoscale flag. A full-scale value of 500 microvolts is suitable for sEMG signals acquired with 10-20 mm wide electrodes. Fig. (5) shows an example of the effect of both autoscale and correct $y$-axis full scale setting on real gait data.

\section{THE POWER SPECTRUM}

A useful tool for qualitative sEMG analysis, derived from statistical signal processing and physics, is the power spectrum plot. It provides a "translation" of the signal in the so called frequency domain. All technical and mathematical details on power spectrum computation, along with its limits, are far from the topics of this paper and will not be discussed here. In brief, the power spectrum of the sEMG signal has a specific belly-shaped profile, while noise, motion artifacts and power line interference have a completely different power spectrum. Thus, before interpreting a signal, one could check for data quality by computing the spectrum. This approach is useful when a continuous trace is detected that could be either a low amplitude continuous muscle activity or just a noisy baseline or an external interference. Fig. (6) can be used as a legend to assess sEMG signal quality in frequency domain.

\section{DATA FILTERING}

Once raw data have been displayed, filters can be used to enhance their aspect (aesthetic) by removing or reducing motion artefact, if any, and baseline slow variations. Normally, if the skin has been properly prepared and cables have been fixed, there is no need for filtering.

Depending on the vendor software, different kind of filters can be selected, such as Butterworth filters, Chebyshev filters and so on. For sEMG application, there is no real practical difference between the available filter technologies. The main parameter to be set is the cut-off frequency, which is usually set at 10 or $20 \mathrm{~Hz}$ to remove slow-varying drifts and potentials.

We again suggest that it is best to look at raw data before filtering, to avoid the risk of misinterpreting data as those presented in Fig. (7).

\section{DATA REPRESENTATION}

Looking at raw data is always the better choice for clinical usage of sEMG data. Rectified EMG is also used to save space on the screen or on the paper. In rectified EMG, negative values are simply forced to be positive, by changing their sign. Rectified data can be also low-pass filtered to provide the EMG envelope. Being smoothed, the envelope appears nicer than raw data (Figs. 8, 9)

For clinical purposes, there is no need to compute the envelope. Conversely, the envelope is used when data 
a)

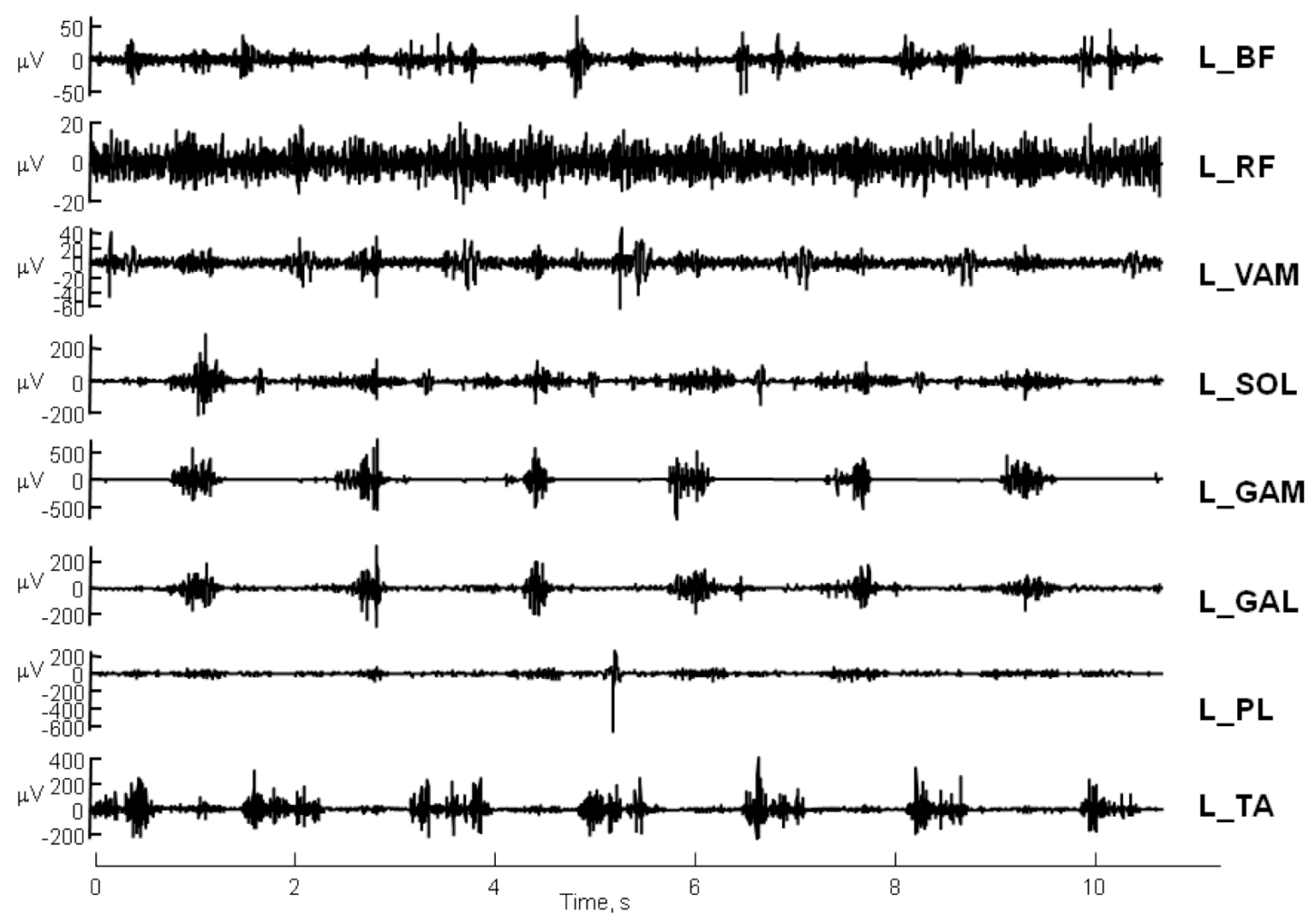

b)

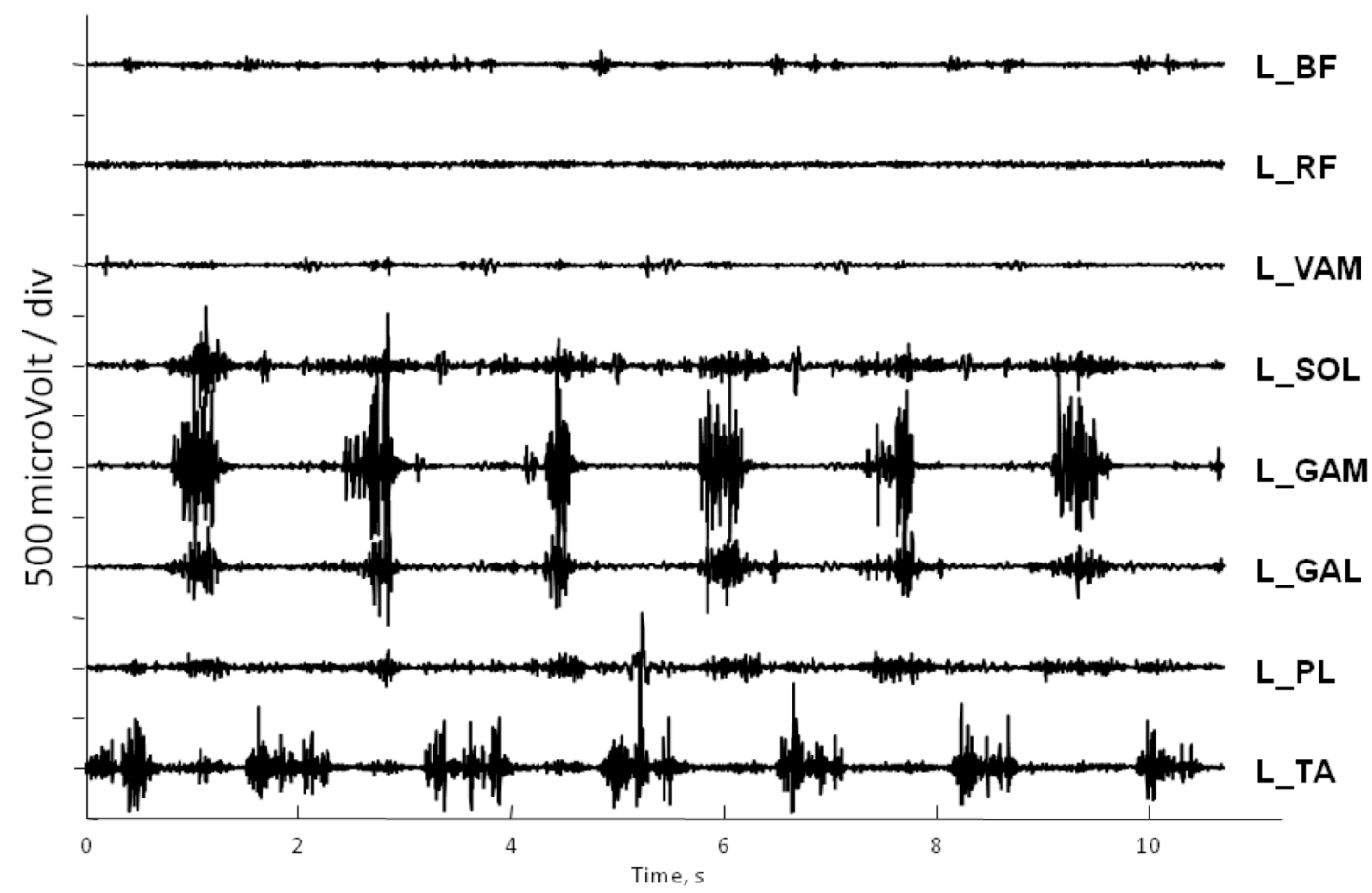

Fig. (5). sEMG data acquired from a healthy adult during gait. The same data are presented in autoscale in a) and, as recommended, with a fixed y-axis full-scale in b). Autoscale dramatically alters data appearance. Based on the auto-scaled representation, rectus femoris seems to have a continuous activity, while it is inactive. Left side (L) muscles. Biceps Femoris (BF), Rectus Femoris (RF), Vastus Medialis (VAM), Soleus (SOL), Gastrocnemius Medialis (GAM), Gastrocnemius Laterali (GAL), Tibialis Anterior (TA). Electrode size: 18 mm, interelectrode distance: $20 \mathrm{~mm}$. 

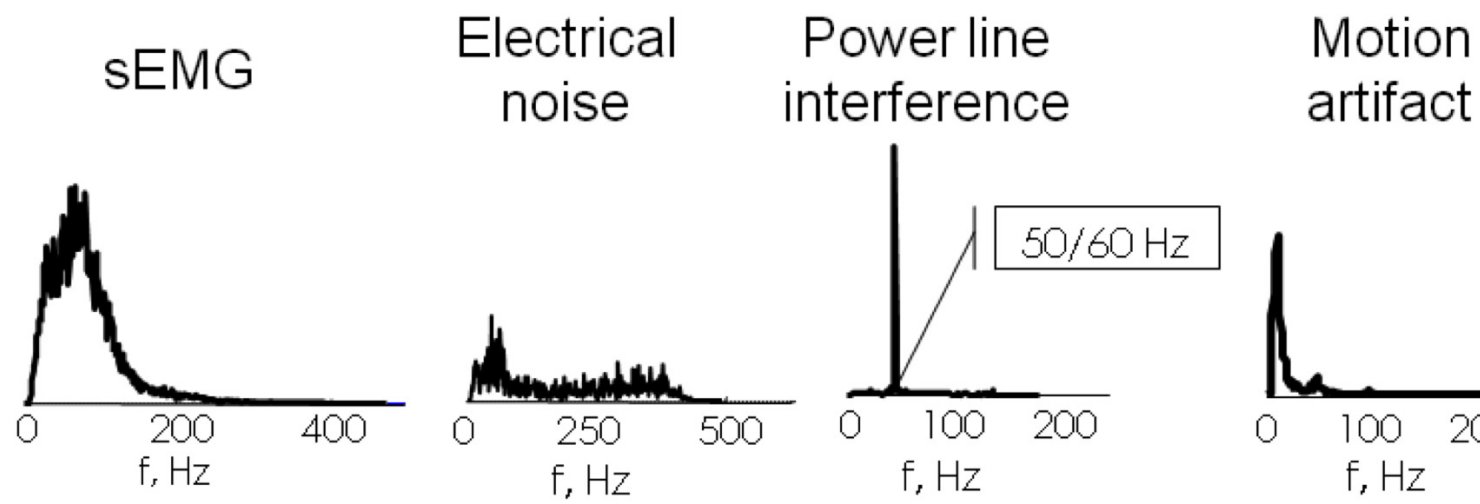

Fig. (6). Power spectrum of the sEMG signal and of the main disturbs that can be present in a signal. From a qualitative point of view, sEMG has a belly-shaped power spectrum, which is completely different from those of noise, power line interference and motion artifact.

\section{RAW DATA}
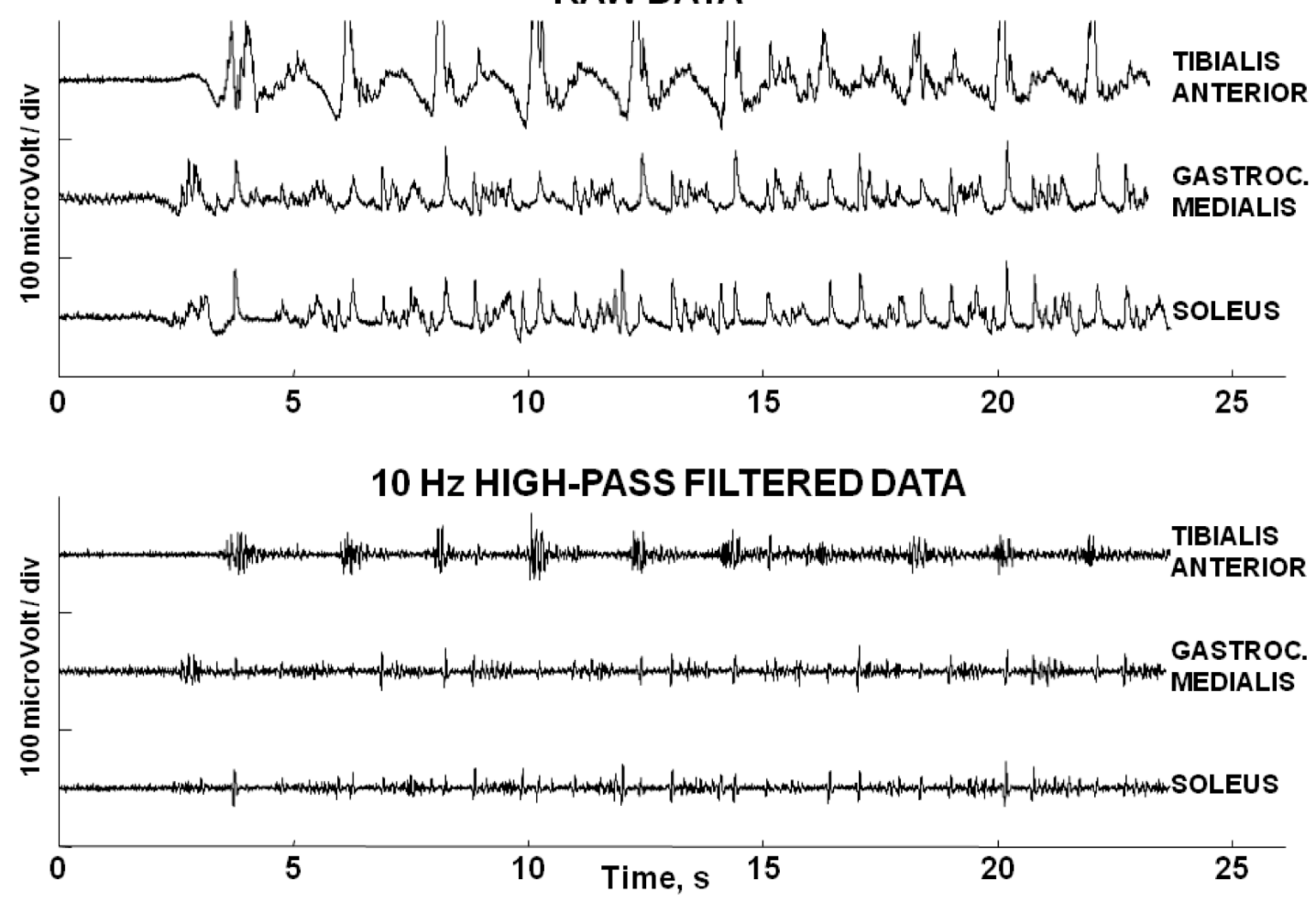

Fig. (7). A borderline case of a meaningless signal, made out by rhythmic artifacts only, that could be misinterpreted as muscle activity after the application of a high pass filter, set at $10 \mathrm{~Hz}$ in this case. Filters are useful to remove minor artifacts and drifts, but raw data should be always checked before filtering.

acquired from different subjects of a homogeneous sample are averaged to obtain an average activation profile. In this case, data are previously normalized in amplitude with respect to a maximum voluntary contraction (MVC).

\section{NUMERICAL INDICATORS}

Numerical indicators are computed based on the differential EMG signal to indicate when muscles are activated during tasks, and to what extent.

As it comprises both a positive and negative signal, the mean amplitude of each EMG signal is nearly zero, despite the activation level. To get a realistic mean value, the signal has to be either rectified or squared before computing the average. The average value of the rectified signal, or ARV, is used as an amplitude indicator. Similarly, the square root of the average value of the squared signal, or RMS, is used as amplitude indicator. Both ARV and RMS are expressed in the same units of the signal (Fig. 10). It is important to outline that, except for controlled isometric conditions, amplitude indicators do not directly reflect either the force produced by the muscle at the tendon or the effect of this force on the joint. The internal net joint moment depends on the resultant of all pulling forces multiplied by their lever arms and the presence of passive mechanical constraints, such as soft tissue contractures, in particular muscle shortening and joint retraction.

Muscle onset estimate deals with the automatic and robust detection of the part of the signal that overcomes the 


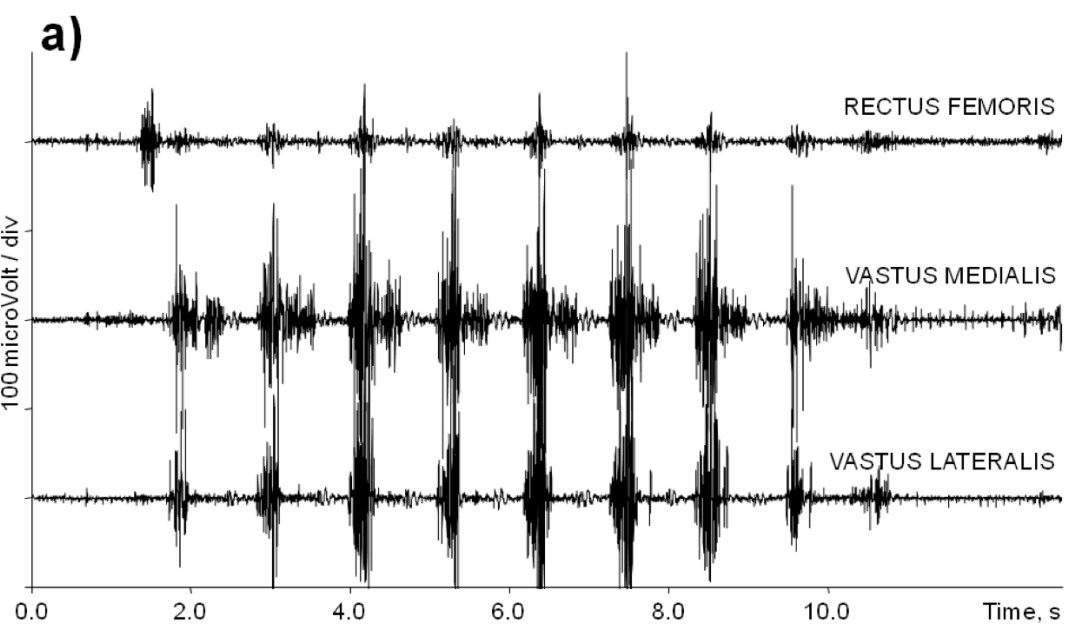

b)

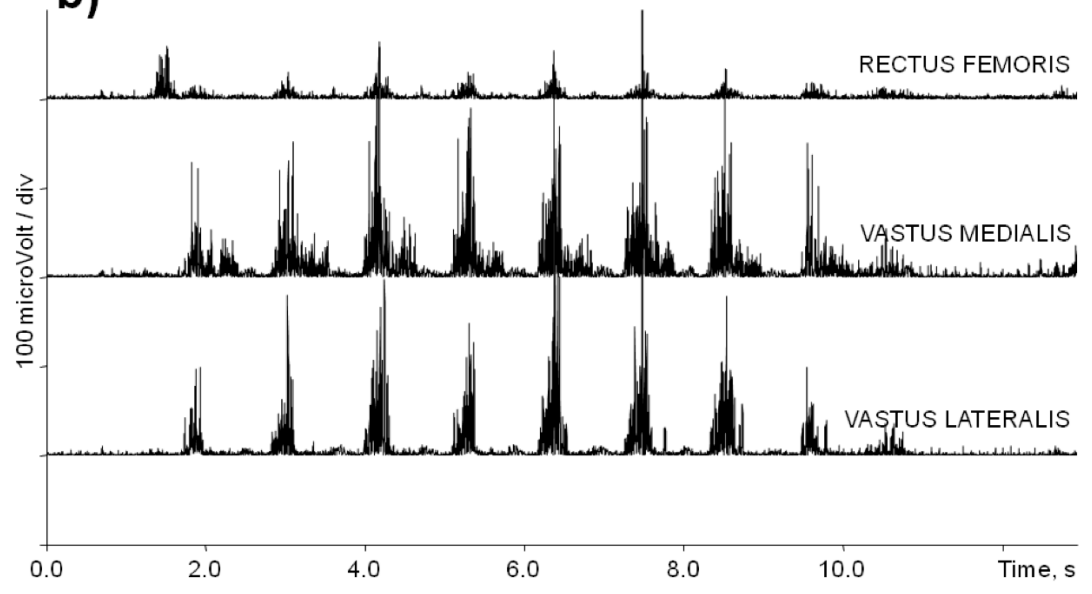

c)

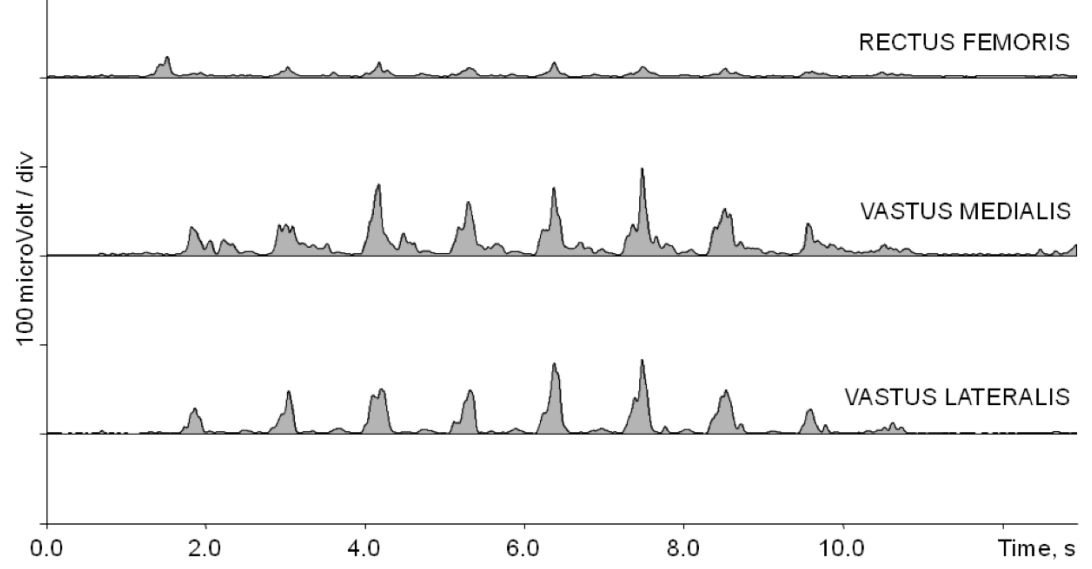

Fig. (8). Different representations of the sEMG signal: raw data (a), rectified data (b), and envelope (c). Rectification turns negative values into positive; the envelope is computed by low-pass filtering the rectified data. A $10 \mathrm{~Hz}$ low pass filter was used in this example.

baseline. Several methods have been developed, with different levels of complexity. In single threshold methods, onset time is defined as the first instant when the rectified signal definitively exceeds a fixed threshold, whose value is based on a previous estimate of the noise level. Such methods provide accurate results when the off-on transition is abrupt or when the ratio between EMG amplitude and noise (baseline) amplitude is high. When the muscle activation occurs with a progressive recruitment of motor units, statistically based detection methods [18, 19] or methods based on the morphological identification of motor unit action potentials [20] should be preferred. 


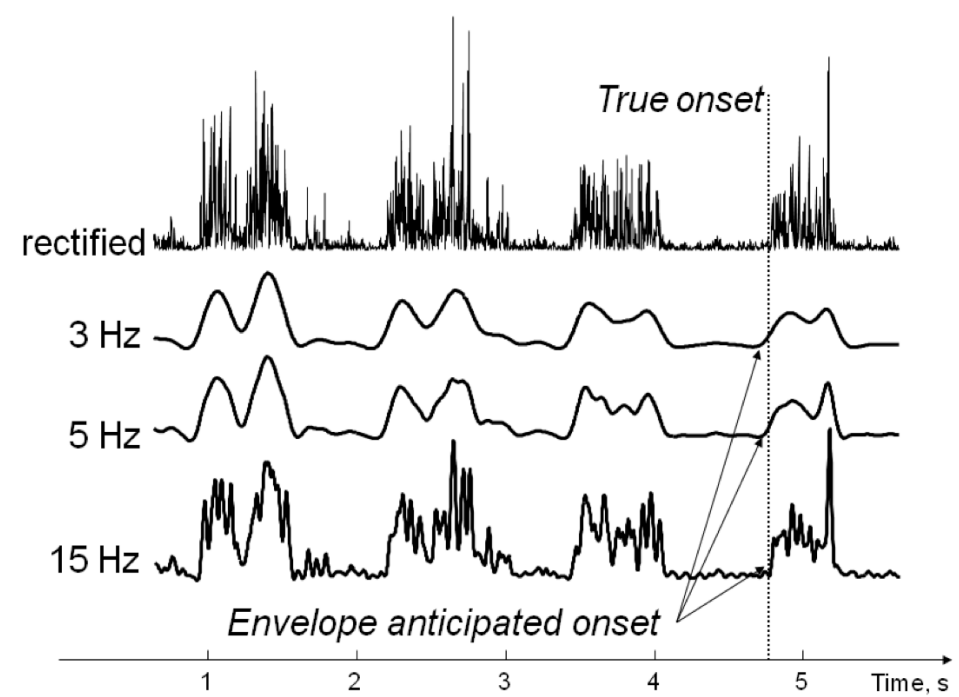

Fig. (9). Effect of the low pass frequency value used to computed the sEMG envelope on the detected muscle onset.

Along with time and amplitude estimators, in the motion analysis field, recent attempts have been aimed at providing a unique indicator that could describe both the presence of muscle activity and its complexity, that is a coarse estimation of the number of recruited motor units [21, 22]. Such estimators could provide clinicians with information on the level of muscle recruitment to a greater extent than would be provided by the amplitude.

\section{FACTORS THAT INFLUENCE SEMG AMPLITUDE}

A complete list of factors that influence sEMG amplitude can be found in [23]. Along with the thickness of the subcutaneous layers, the electrode area and the interelectrode distance, amplitude variations may occur during movements because of the shift of the muscle fibers with respect to the electrodes on the skin. If the sources move away from the detecting system, the amplitude will decrease. Conversely, amplitude is increased when sources move towards the electrodes.

\section{MUSCLES THAT CAN BE MEASURED WITH SMALL ELECTRODES}

Small size electrodes (e.g. $2 \mathrm{~mm}$ in diameter) are preferable for sEMG detection due to their selectivity. Being selective, small electrodes provide low amplitude potentials, thus requiring proper amplification or $\mathrm{A} / \mathrm{D}$ resolution. Furthermore, skin-electrode impedance has to be reduced by mild skin rubbing. The main advantage of using small electrodes is the possibility of detecting sEMG from almost all superficial muscles. We report here a list of the muscles from which sEMG was accurately acquired by using $1 \mathrm{~mm}-$ wide electrodes, as presented in [24], divided by anatomical district. Shoulder: upper trapezius, middle trapezius, lower trapezius, anterior deltoideus, posterior deltoideus, rhomboideus minor, rhomboideus major, infraspinatus, teres major. Back, Abdomen and Neck: sternocleidomastoid sternal head, sternocleidomatoid clavicular head, anterior scalenus, pectoralis major, obliquus externus abdominis, latissimus dorsi, erector spinae, serratus anterior, rectus abdominis. Arm and Hand: triceps brachii lateral and medial head, biceps brachii long and short head, brachioradialis, anconeus, abductor pollicis brevis, opponens pollicis, pronator teres, Palmaris longus, abductor digiti minimi. Lower Limb: gluteus medius, gluteus maximus, tensor fasciae latae, rectus femoris, semitendinosus, vastus medialis, vastus lateralis, biceps femoris, gastrocnemius medialis and lateralis, tibialis anterior, soleus, peroneus longus, peroneus brevis.

\section{INFORMATION PROVIDED BY sEMG}

The easiest information that can be obtained by a sEMG investigation is if a muscle is active or not during a voluntary task or after a passive manoeuvre (e.g. a quick stretch). The agonist-antagonist recruitment strategy can be studied, outof-phase overactive muscle can be detected in neurologic patients, tremors and tremor frequency can be assessed, and so on.

In general, clinicians can gain knowledge from three main characteristic of the sEMG signal: timing, morphology and - with the discussed limitations - amplitude. The book Muscle Alive [25] can be a reference for all those who want to approach sEMG.

\section{FACTORS THAT MAY AFFECT SEMG TIMING}

Timing is measured by means of onset detection methods, as described above, as expressed in percentage of the task duration, rather than in seconds. Muscle activity intervals during gait are expressed in percentage of the gait cycle (\%GC). For instance, soleus is active from $15 \% \mathrm{GC}$ to $50 \% \mathrm{GC}$ during spontaneous level walking of healthy subjects. Normative data can be found in [26]. While analyzing patients, the amount of timing alteration can be in the order of several percentage points of the cycle, thus being detectable by visual inspection. In the gait of patients with neurological lesion, for instance, a muscle can be activated out of phase, that is during swing instead of in stance [27].

Factors that can alter the sEMG trace will also alter the on/off automatic analysis. Thus, the noise level should be kept low by a proper skin preparation, the presence of motion artefact has to be checked and the possibility of 

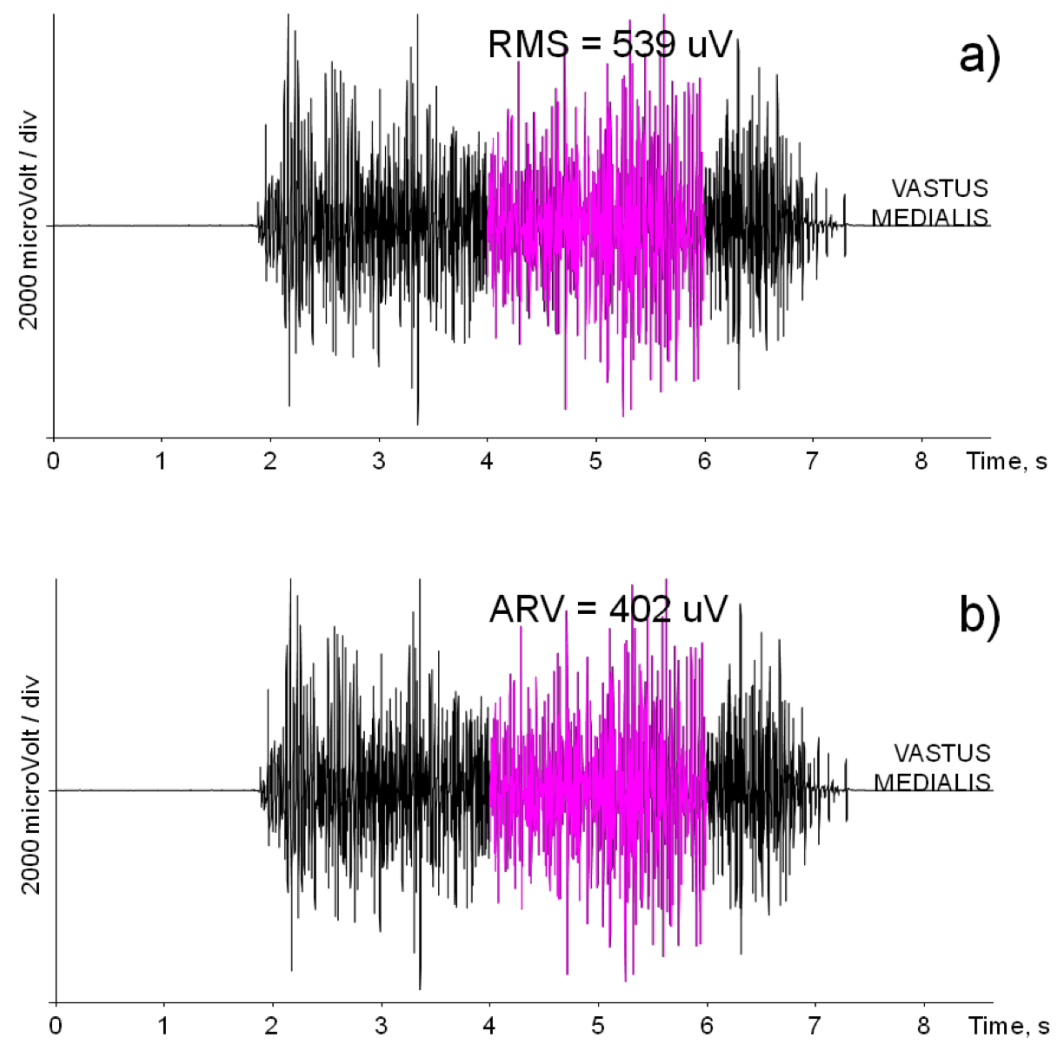

Fig. (10). EMG amplitude estimators. Root mean square (RMS) and average rectified value (ARV) computed on a selected epoch of the sEMG signal.

crosstalk has to be addressed by means of preliminary operator-driven testing contractions.

Muscle on-off is typically reported as a percentage range within a cycle. Thus, an error in the identification of either the beginning or the end of the cycle events will alter the onset values. For instance, a delay in the identification of the foot-ground contact during gait, which defines the gait cycle, will result in anticipated values for all the detected muscle onsets.

Other than technical errors, muscle timing may also be influenced by subject-specific factors such as walking speed, pain, limb rotation with respect to the direction of the movement, muscle overactivity, altered recruitment, muscle shortening.

Variations in walking speed primarily affect the sEMG amplitude, rather then timing [28]. However, extreme reductions in walking speed cause changes in locomotor task demands thus altering the muscle timing. In this case, normative data on muscle onset obtained from healthy subject walking at self-selected speed are not a proper reference. To provide reference values, spontaneous gait has a speed, normalized to subject height, of about $75 \%$ height $/ \mathrm{s}$ $-85 \%$ height/s and gait turns into a sequence of postures at about 30\% height/s [28, 29].

Pain can alter the sEMG timing. Muscle pattern can be completely altered to prevent pain and, according to the patient pathology, this factor has to be kept in mind before any inferences are made. For instance, muscle activity of masticatory muscles is affected by dental malocclusions and the chewing pattern is adapted to allow for a painless closure [30].

Limb rotation with respect to the direction of movement may alter the functional effect of muscles and lever arms. Thus, muscle onset will depend on the new biomechanical configuration. Such effect can be easily reproduced by asking a healthy subject to walk with an externally rotated limb and measuring the sEMG activity of both gastrocnemius medialis and lateralis.

Muscle overactivity is one of the positive signs in upper motor neuron syndrome (UMNS). Muscle timing can be greatly altered in these patients, being absent, shortened, prolonged, out of phase or continuous. Again, muscle can be trigged by fast stretches, as in the case of spasticity. The use of sEMG during gait, and during movement in general, provide useful information in the planning of the treatment for patients with UMNS. In fact, while spasticity, defined as in [31], is frequently found on clinical examination with the subject at rest, it rarely appears during the patients slow gait, in which fast joint rotations do not occur. To better understand sEMG data in UMNS patients, several tasks can be acquired, suited to enhance the underlying phenomena. For instance, gait trials at maximum walking speed can be acquired and used to allow for a differential analysis of abnormal muscle patterns.

Muscle shortening alters the muscle onset because of the mechanical constraint introduced. For instance, in patients who have had a stroke, plantarflexor muscles may tend to shorten progressively. In some of these patients the timing of 
the plantarflexors, becomes shorter, irregular and may disappear.

\section{FACTORS THAT MAY AFFECT SEMG MORPHO- LOGY}

sEMG morphology can be defined as the shape of the trace. It is a qualitative aspect of sEMG, which is observed by visual inspection. Morphology deals with 1) the recruitment modality of a muscle, which is normally gradual but can be abrupt in patients, 2) the presence of separate bursts instead of a unique modulated activity, 3) the level of interference in the trace, which is due to the summation of muscular sources and can be reduced in the case of paucity of active motor units.

Being related to the shape of the detected signal, morphology may be altered by all those factors that may increase or smooth the sEMG trace, such as the electrode size, the interelectrode distance, the distance between the source and the detection system and both hardware and software filters.

\section{FACTORS THAT MAY AFFECT SEMG AMPLITUDE}

sEMG amplitude is the level, in microvolts, reached by the trace during the task. It may be thought by clinicians approaching sEMG that an increase in amplitude would result in a proportional increase of the mechanical effect of the active muscle on the joint it crosses. sEMG amplitude, typically expressed by the RMS value computed on consecutive epochs, has a linear relationship with force in isometric contractions only for certain muscles as reviewed in [16]. During gait and generally during movement, this relationship fails. As described above, the mechanical effect of muscle activation on a joint depends on the balance between the internal and the external moment and the internal joint moment depends on the resultant of all pulling forces multiplied by their lever arms and the presence of passive mechanical constraints, such as soft tissue contractions. sEMG amplitude is strongly affected by a long list of anatomical and technical factors, such as fibre size, depth, thickness of subcutaneous layers, electrode size and interelectrode distance. A complete list of factors that affect sEMG amplitude can be found in [23]. Furthermore, the mechanical effect of a muscle contraction strictly depends on both the length-tension and tension-velocity curves, which are altered, for instance, by muscle shortening and muscle stiffness [32].

In the assessment of patients with neurological disease, we do not interpret sEMG amplitude values but look at the presence of amplitude variation, which may account for the ability of a muscle to vary its contraction level in response to a variation in the mechanical demand.

When maximum voluntary contractions can be performed by subjects, as in studies on healthy subjects and athletes, MVC normalised RMS amplitude values can be used ad indicators of force in strictly controlled task [16].

\section{CONCLUSION}

This work describes the process of sEMG acquisition, from the source to the screen, along with a summary of the signal variables that can be used by clinicians, and focuses on the factors that may alter the detected signal and that may lead to misinterpretation. Despite the long list, most of the error sources can be avoided by treating the skin, using small electrodes with adequate adhesion, placing the electrodes according to protocols and representing raw data on a fixed $\mathrm{y}$-axis scale.

\section{REFERENCES}

[1] Merletti R, Parker PA. Electromyography: Physiology, Engineering and Noninvasive Applications. Hoboken, USA: IEEE Press and John Wiley \& Sons 2004.

[2] Disselhorst-Klug C, Silny J, Rau G. Improvement of spatial resolution in surface-EMG: a theoretical and experimental comparison of different spatial filters. IEEE Trans Biomed Eng 1997; 44(7): 567-74.

[3] Merletti R, Botter A, Troiano A, Merlo E, Minetto MA Technology and instrumentation for detection and conditioning of the surface electromyographic signal: state of the art. Clin Biomech (Bristol, Avon) 2009; 24(2): 122-34.

[4] Farina D, Cescon C. Concentric-ring electrode systems for noninvasive detection of single motor unit activity. IEEE Trans Biomed Eng 2001; 48(11): 1326-34.

[5] Merletti R, Farina D, Gazzoni M, Merlo A, Ossola P, Rainoldi A. Surface electromyography: A window on the muscle, a glimpse on the central nervous system. Europa Medicophysica 2001; (37): 5768.

[6] Drost G, Stegeman DF, van Engelen BG, Zwarts MJ. Clinical applications of high-density surface EMG: a systematic review. J Electromyogr Kinesiol 2006; 16: 586-602.

[7] Blumenstein R, Basmajian J. Electrode placement in EMG biofeedback. Baltimore, USA: Williams \& Wilkins 1980.

[8] Hermens H, Freriks B, Disselhorst-Klug C, Rau G. Development of recommendations for SEMG sensors and sensor placement procedures. J Electromyogr Kinesiol 2000; 10: 361-74.

[9] Hermens HJ, Freriks B, Merletti R, et al. Eds. SENIAM 8: European recommendations for surface electromyography, ISBN: 90-75452-15-2. The Netherlands: Roessingh Research and Development bv 1999.

[10] Bottin A, Rebecchi P. Impedance and noise of the skin-electrode interface in surface EMG recordings. In: Proc XIV Congr Int Soc Electrophysiol Kinesiol Wien 2002; 246-7.

[11] Webster JG. Medical Instrumentation: Application and Design. New York, USA: John Wiley \& Sons Inc. 1995.

[12] Tam H, Webster JG. Minimizing electron motion artifact by skin abrasion. IEEE Trans Biomed Eng 1977; 31: 823-6.

[13] Campanini I, Merlo A, Degola P, Merletti R, Vezzosi G, Farina D. Effect of electrode location on EMG signal envelope in leg muscles during gait. J Electromyogr Kinesiol 2007; 17(4): 515-26.

[14] Blanc Y. Surface electromyography (SEMG): a plea to differentiate between crosstalk and co-activation. In: Hermens HJ, Freriks B, Eds. The state of the art on sensors and sensor placement procedures for surface electromyography: a proposal for sensor placement procedures deliverable, 5 of the SENIAM project. The Netherlands: Roessingh Research and Development bv 1997; 96100 .

[15] Johanson ME, Radtka SA. Amplitude threshold criteria improve surface electrode specificity during walking and functional movements. Gait Posture 2006; 24(4): 429-34.

[16] Disselhorst-Klug C, Schmitz-Rode T, Rau G. Surface electromyography and muscle force: limits in sEMG-force relationship and new approaches for applications. Clin Biomech (Bristol, Avon) 2009; 24(3): 225-35. Review.

[17] Merletti R, Hermens H. Detection and conditioning of the surface EMG signal. In: Merletti R, Parker PA, Eds. Electromyography: Physiology, Engineering and Noninvasive Applications. Hoboken, USA: IEEE Press and John Wiley \& Sons 2004; pp. 107-28.

[18] Bonato P, Alessio TD, Knaflitz M. A statistical method for the measurement of muscle activation intervals from surface myoelectric signal during gait. IEEE Trans Biomed Eng 1998; 45: 287-99.

[19] Staude G, Wolf W. Objective motor response onset detection in surface myoelectric signals. Med Eng Phys 1999; 21: 449-67.

[20] Merlo A, Farina D, Merletti R. A fast and reliable technique for muscle activity detection from surface EMG signals. IEEE Trans Biomed Eng 2003; 50: 316-23. 
[21] Li X, Zhou P, Aruin AS. Teager-Kaiser energy operation of surface EMG improves muscle activity onset detection. Ann Biomed Eng 2007; 35(9): 1532-8.

[22] Ahmad SA, Chappell PH. Surface EMG pattern analysis of the wrist muscles at different speeds of contraction. J Med Eng Technol 2009; 33(5): 376-85.

[23] Farina D, Merletti R, Enoka RM. The extraction of neural strategies from the surface EMG. J Appl Physiol 2004; 96(4): 1486-95.

[24] Nannucci L, Merlo A, Merletti R, et al. Atlas of the innervation zones of upper and lower extremity muscles. Proceedings of the XIV ISEK Congress, Vienna 2002; 353-4.

[25] Basmajian JV, De Luca C. Muscles Alive - Their Functions Revealed by Electromyography, $5^{\text {th }}$ ed. Baltimore, USA: Williams \& Wilkins 1985.

[26] Sutherland DH. Gait disorders in childhood and adolescence. Baltimore, USA: Lippincott Williams \& Wilkins 1984.

[27] Blanc Y. EMG timing errors of pathologic gait. In: Hermens HJ Ed. Proceedings of the first general SENIAM (surface EMG for non-invasive assessment of muscles) workshop, Torino, Italy. Enschede, The Netherlands: Roessingh Research and Development 1996; pp. 183-5.

[28] Den Otter AR, Geurts AC, Mulder T, Duysens J. Speed related changes in muscle activity from normal to very slow walking speeds. Gait Posture 2004; 19(3): 270-8.

[29] Campanini I, Merlo A. Reliability, smallest real difference and concurrent validity of indices computed from GRF components in gait of stroke patients. Gait Posture 2009; 30(2): 127-31.

[30] Piancino MG, Farina D, Talpone F, Merlo A, Bracco P. Muscular activation during reverse and non-reverse chewing cycles in unilateral posterior crossbite. Eur J Oral Sci 2009; 117(2): 122-8.

[31] Lance JW. Symposium synopsis. In: Feldman RG, Young RR, Koella WP, Eds. Spasticity: disordered motor control. Chicago, USA: Yearbook Medical 1980; 485-94.

[32] Craik RL, Oatis CA. Gait Analysis: Theory and Application. St. Louis, USA: Mosby Inc. 1995.

(C) Merlo and Campanini; Licensee Bentham Open

This is an open access article licensed under the terms of the Creative Commons Attribution Non-Commercial License (http: //creativecommons.org/licenses/by$\mathrm{nc} / 3.0 /$ ) which permits unrestricted, non-commercial use, distribution and reproduction in any medium, provided the work is properly cited. 